\title{
Redes neuronales artificiales para la predicción de flujos de carga aplicadas al sistema de trasmisión de Uruguay
}

\section{Artificial neural networks for the prediction of power flows applied to the Uruguayan transmission system.}

Santiago Garabedian 1 , Rodrigo Porteiro², Pablo Pena ${ }^{3}$

Garabedian, S; Porteiro, R; Pena, P. Redes neuronales artificiales para la predicción de flujos de carga aplicadas al sistema de trasmisión de Uruguay. Tecnología en Marcha. Vol. 34, especial. ALTAE. Diciembre 2021. Pág 182-192.

doi) https://doi.org/10.18845/tm.v34i7.6040

1 Instituto de Ingeniería Eléctrica, Facultad de Ingeniería, Universidad de la República. Administración Nacional de Usinas y Transmisiones Eléctricas, UTE, Uruguay. Correo electrónico: sgarabedian@ute.com.uy

2 Instituto de Ingeniería Eléctrica, Facultad de Ingeniería, Universidad de la República. Administración Nacional de Usinas y Transmisiones Eléctricas, UTE, Uruguay. Correo electrónico: rporteiro@ute.com.uy

3 Instituto de Ingeniería Eléctrica, Facultad de Ingeniería, Universidad de la República. Administración Nacional de Usinas y Transmisiones Eléctricas, UTE, Uruguay. Correo electrónico: ppenaV@ute.com.uy 


\title{
Palabras clave
}

Flujos de carga; redes neuronales.

\section{Resumen}

En el presente trabajo se propone el uso de redes neuronales artificiales para la resolución del problema de flujos de carga. El estudio del flujo de carga de la red eléctrica constituye una herramienta fundamental para la operación y la planificación de un sistema eléctrico. En términos matemáticos el problema de flujos de carga se resuelve a través de un sistema de ecuaciones no lineales. Para dicha resolución tradicionalmente se han utilizado diversos métodos numéricos, principalmente el método de Newton-Raphson y sus variantes. Estos métodos numéricos aplicados a sistemas eléctricos de gran tamaño tienen un alto costo computacional asociado. Resolver una cantidad considerable de flujos de carga utilizando estos métodos implica incurrir en tiempos de ejecución que resultan prohibitivos en estudios sobre la red eléctrica. Este problema se vuelve crítico en estudios de casos de contingencia, incluso utilizando el criterio simple de contingencia $N$-1. La construcción de redes neuronales que aproximen la resolución de flujos de carga permite reducir significativamente los tiempos de ejecución de los estudios anteriormente mencionados. En este trabajo se propone el diseño de una arquitectura de red neuronal para la aproximación de flujos de carga. Utilizando la arquitectura diseñada se implementa un modelo de aproximación de flujos de carga. La validación de la herramienta se realiza utilizando la red de trasmisión de Uruguay. La aproximación obtenida para este caso de estudio es evaluada aplicando la métrica MAPE y se obtiene un valor de $2.6 \%$, lo que constituye un resultado muy prometedor.

\section{Keywords}

Power flow; neural networks.

\begin{abstract}
In the present work, the use of artificial neural networks is proposed to solve the load flow problem. The study of the load flow of the electrical network constitutes a fundamental tool for the operation and planning of an electrical system. The load flow problem is solved by a system of non-linear equations. For this resolution, numerical methods have traditionally been used, mainly the Newton-Raphson method and its variants. These numerical methods applied to large electrical systems are very expensive in terms of computational cost. Solving a considerable number of load flows using these methods involves incurring in execution times that are prohibitive in studies of the electrical network. This problem becomes critical in contingency case studies, even using the simple $\mathrm{N}-1$ contingency criterion. The construction of neural networks that approximate the resolution of load flows allows to significantly reducing the execution time of the aforementioned studies. In this work, the design of a neural network architecture for the approximation of load flows is proposed. Using the designed architecture, a load flow approximation model is implemented. The validation of the tool is carried out using the Uruguayan transmission network. The approximation obtained for this case study is evaluated by applying the MAPE metric and a value of $2.6 \%$ is obtained, which constitutes a very promising result.
\end{abstract}




\section{Introducción}

El análisis de flujos de carga en régimen estacionario constituye una herramienta fundamental para el análisis de un sistema eléctrico, tanto en la etapa de operación, como en la de planificación [1]. Esta herramienta es aplicada tanto a nivel de trasmisión como a nivel de distribución con el fin de evitar problemas de funcionamiento, ya sea por sobrecarga de elementos o tensiones inadmisibles. El análisis de distintos escenarios críticos a través de esta técnica es crucial a la hora de tomar decisiones con incertidumbre. Históricamente, un conjunto acotado de escenarios extremos seleccionados cuidadosamente por expertos era analizado.

Hoy en día, la topología de la red eléctrica es cada vez más compleja (mayor cantidad de configuraciones posibles). A su vez, el sistema de generación ha pasado de estar localizado en unos pocos nodos a estar cada vez más distribuido. Además, la fuerte inserción de generación renovable constituye un desafío en cuanto a la variabilidad del recurso asociado a la generación, como sucede con las fuentes eólica y solar [2].

Por estas razones, es necesario analizar un conjunto más extenso de escenarios (de ahora en más casos) y resulta imposible que lo defina un experto.

El problema de flujos de carga es resuelto mediante un sistema de ecuaciones no lineales. Para la resolución computacional de este tipo de sistemas existen metodologías basadas en métodos numéricos iterativos. La principal técnica es el método de Newton-Raphson y sus variantes, analizados en profundidad en los trabajos de Stott[3], Uemura[4], Peterson[5] y Tinney[6,7]. Cualquiera de estos métodos tiene el inconveniente de incurrir en grandes costos computacionales, lo que limita el análisis de un conjunto extenso de casos. Una alternativa razonable es utilizar una técnica de aproximación lineal conocida como Flujo DC como en el trabajo de Stott[8]. Esta técnica asume determinadas hipótesis en las características de los elementos de la red que permiten convertir el problema en la resolución de un sistema lineal de ecuaciones. Esto reduce sensiblemente el tiempo de resolución. Si bien la resolución mediante el Flujo DC brinda una buena aproximación a la solución del problema, tiene limitaciones con respecto al análisis de ciertos problemas, en particular análisis de tensiones y pérdidas, que son esenciales para el correcto desempeño de la red.

\section{Resolución del problema de flujo de carga}

En esta sección se presenta la formulación matemática del problema de flujo de carga y luego el abordaje tomado en este trabajo mediante redes neuronales que aproximan dicho problema.

\section{Consideraciones Generales}

El problema del flujo de carga se basa en obtener el voltaje en magnitud y fase de cada nodo de la red, a partir de los cuales quedan determinadas el resto de las variables, como ser el flujo por los elementos. Debido a que se trata de un problema que no es lineal ni convexo, no existen técnicas analíticas de resolución exacta, y debe resolverse mediante métodos numéricos iterativos. El problema se puede plantear a través de las siguientes ecuaciones, una correspondiente a la potencia activa (Ec. 1) y otra correspondiente a la reactiva (Ec. 2) para cada uno de los nodos de la red.

$$
0=-P_{i}+\sum_{k=1}^{N}\left|V_{i}\right|\left|V_{k}\right|\left(G_{i k} \cos \theta_{i k}+B_{i k} \sin \theta_{i k}\right)
$$




$$
0=-Q_{i}+\sum_{k=1}^{N}\left|V_{i}\right|\left|V_{k}\right|\left(G_{i k} \sin \theta_{i k}-B_{i k} \cos \theta_{i k}\right)
$$

donde $P_{i}$ y $Q_{i}$ son las potencias activas y reactivas respectivamente, las variables $V$ y $\theta$ representan el voltaje en magnitud y fase. Los parámetros $G$ y $B$ son propiedades físicas de las líneas.

De las variables anteriores se sabe lo siguiente:

- $G, B$ y la potencia activa inyectada son datos de entrada

- Los ángulos $\theta$ son desconocidos salvo en el/los nodo/s slack, donde la fase es impuesta en el valor cero. Existe al menos un nodo slack.

- La potencia reactiva puede ser conocida en los nodos donde el generador es capaz de controlar el voltaje regulando la reactiva (dentro de ciertos límites). En cualquier otro caso es una variable desconocida.

\section{Redes neuronales y formulación del problema}

En los últimos años las técnicas de aprendizaje automático, en particular el aprendizaje profundo, se han utilizado cada vez con más frecuencia en diversos campos de la ciencia. Los métodos numéricos tradicionales aplicados a la resolución de problemas no se clasifican como métodos de aprendizaje, sino como métodos exactos. Los métodos de aprendizaje se pueden entender como cajas negras con ciertos parámetros que aproximan una función desconocida. De la función se conoce un conjunto de salidas correspondientes a un conjunto determinado de entradas. Los parámetros se ajustan de forma que el conjunto de salidas del modelo generado a partir del conjunto de entradas ajuste a la salida real. Dicho ajuste se realiza minimizando el error asociado a una métrica en particular que compara la salida real con la obtenida del modelo.

Entre las técnicas de aprendizaje automático, las redes neuronales profundas han demostrado grandes resultados para la resolución de problemas muy complejos, lo que ha motivado el abordaje de este trabajo. Se destacan en este sentido las áreas del reconocimiento de imágenes y del procesamiento del lenguaje natural.

El uso de técnicas de aprendizaje automático para aproximar el flujo de carga presenta un gran desafío a la hora de entrenar el modelo. Sin embargo, tiene la ventaja que una vez que se obtienen los

parámetros de la red neuronal asociada, el cálculo de un flujo de carga se reduce a la aplicación de simples funciones matemáticas encadenadas. Esto reduce notablemente los costos computacionales asociados a las técnicas citadas en la sección 1, sobre todo cuando se debe aplicar a gran cantidad de casos en el marco de un estudio de planificación de la expansión de la red.

La idea principal de este trabajo es entrenar una red neuronal para la predicción de flujos en el sistema eléctrico de Uruguay a nivel de trasmisión ( 150 kV y 500 kV). 


\section{Trabajos relacionados}

Muchos trabajos se han realizado en años recientes aplicando técnicas de aprendizaje automático a la resolución de problemas relacionados con el sector eléctrico. Diversos estudios que utilizan algoritmos de aprendizaje automático se han realizado para monitorear la estabilidad del voltaje en la red eléctrica, como por ejemplo el estudio de Malbas[9]. Estos trabajos se basan en realizar predicción de ciertas variables basada en datos medidos.

Otra de las ramas de la ingeniería eléctrica en dónde se han aplicado técnicas de aprendizaje automático es la de detección de fallas en las líneas de trasmisión, los trabajos de Chen [10] y Hasan [11] se destacan entre otros.

Sin embargo, en el problema que abordamos en este artículo son pocos los trabajos realizados utilizando estas técnicas. La mayor parte de estos trabajos apuntan a resolver el problema general del flujo óptimo (OPF) o sus variantes. El trabajo de Pan[12] se enfoca en la aplicación de redes neuronales profundas para la resolución del problema de OPF mientras que los trabajos de Marot [12] y Owerko [14] utilizan otro tipo de arquitecturas de redes neuronales que contemplan la topología en su estructura.

Dos trabajos relevantes aplican técnicas de inteligencia artificial a la resolución del problema de flujo de carga estándar. En el estudio realizado por Paucar [15] se aplican técnicas de perceptrón multicapa en redes eléctricas pequeñas. En un trabajo reciente de Donnot[16], técnicas más complejas de aprendizaje profundo son utilizadas para la resolución de flujos de carga en redes de trasmisión reales de tamaño considerable.

\section{Diseño e implementación de la solución}

En esta sección se describen, el diseño e implementación de la solución propuesta, las etapas de manejo de datos y entrenamiento del modelo asociado. También se presenta la implementación en software de la solución.

\section{Manejo de datos y métrica utilizada}

En esta subsección se describe la preparación y estructura de datos de entrada y salida, así como las métricas utilizadas al realizar la evaluación de resultados experimentales.

\section{Preparación de datos}

Para obtener los datos adecuados para el entrenamiento del modelo desarrollado se resolvieron 40000 casos en la herramienta de Siemens Industry PSS/E[17], obteniéndose un conjunto de resultados que representan flujos por cada elemento de la red. Los datos de generación y demanda para la creación de los distintos escenarios de flujos eléctricos en régimen estacionario se obtuvieron a partir del sistema de simulación SimSE[18].

Los flujos de carga resueltos hasta lograr la convergencia deben cumplir con las tensiones admisibles por nodo impuestas en el software PSS/E (rango desde 0.95 a 1.05), así como también los límites de reactiva que pueden entregar los generadores o compensadores modelados. Los casos que no convergen y los que no cumplen límites de reactiva o tensiones, son descartados para el presente caso de estudio. En la resolución de los flujos de carga, se utilizó la opción de ajuste automático de TAPs y compensación shunt implementada en PSS/E para lograr el ajuste de las tensiones al rango admisible. Esto podría tener inconvenientes a la hora de entrenar el modelo, debido a que las soluciones de casos similares pueden tener ajustes de compensación y TAPs diferentes. Esto se traduce en variaciones relevantes de flujos en los elementos para situaciones de generación y demanda similares, o incluso idénticas. Este efecto es analizado en profundidad en el trabajo de Pena [19]. De la red uruguaya de trasmisión 
se consideraron sólo los elementos de 150 kV y 500 kV. Éste modelado resulta en una red eléctrica con 190 elementos entre líneas aéreas, cables subterráneos y transformadores. En este trabajo se considera una topología dada, basada en el modelo publicado por UTE [20], y no se analizan contingencias.

\section{Descripción de datos}

Los datos de entrada consisten en un vector de potencias asociado a las generaciones y demandas. Los datos de salida son las potencias en MVA de un nodo a otro nodo a través de un elemento que puede ser una línea aérea (de 150 kV o 500 kV), cable subterráneo de 150 kV o transformador.

- Entrada: Potencias de generación y demanda (MW), (199 componentes). Se asigna por convención un signo positivo a la generación y negativo a la demanda.

- Salida: Potencias de flujo de cada elemento (MVA), (380 componentes), se toma para cada flujo el valor desde ambos nodos extremos. Indicando el sentido del flujo con una convención de signo.

\section{Métrica utilizada}

Se utilizó para la evaluación el error absoluto porcentual medio (MAPE), definido en (Ec. 3). En

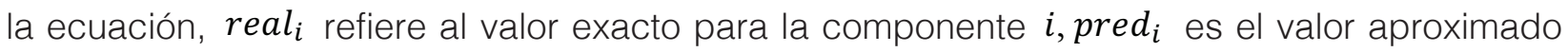
por el modelo, y $n$ el número de componentes de los vectores evaluados.

$$
M A P E=100 \times \frac{\sum_{i=1}^{n}\left|\frac{\text { real }_{i}-\text { pred }_{i}}{\text { real }_{i}}\right|}{n}
$$

Arquitectura de la red neuronal

El diseño de la red neuronal puede describirse mediante el conjunto de ecuaciones 4.

$$
\begin{aligned}
& h_{0}=\vec{P} \\
& h_{1}=\sigma_{1}\left(W_{1} h_{0}+b_{0}\right) \\
& \vec{F}=\sigma_{2}\left(W_{2} h_{1}+b_{1}\right)
\end{aligned}
$$

La capa de entrada $h_{0}$ recibe un vector $\vec{P}$ con las potencias de generación y demanda en la red. En la capa oculta de la red neuronal, $W_{1}$ representa la matriz de pesos y $b_{0}$ el vector de sesgos, mientras $\sigma_{1}$ es la función de activación. En este trabajo para $\sigma_{1}$ se utilizó la función de activación RELU, que introduce el componente no lineal necesario para aproximar la función deseada. La salida de la capa oculta es $h_{1}$. Finalmente, en la capa de salida, $W_{2}$ representa la matriz de pesos y $b_{1}$ el vector de sesgos, mientras es la función de activación. Por ser el rango de la salida el conjunto de los números reales, es una función de activación lineal.

\section{Entrenamiento del modelo}

Una vez que los datos fueron preparados de acuerdo a lo descrito, se lleva a cabo un procedimiento de cuatro etapas para el entrenamiento y la evaluación del modelo. Las cuatro etapas son: 
- Se separan los datos en 3 particiones tomadas al azar en proporciones 4:1:1. Estos conjuntos son: entrenamiento, validacióny testing. El conjunto de entrenamiento es utilizado para ajustar los pesos y sesgos del modelo mediante el algoritmo de backpropagation, estándar para entrenamiento de redes neuronales.

- El conjunto de validación es utilizado para ensayar diferentes juegos de hiperparámetros. Se utilizó con este fin una búsqueda de hiperparámetros en una grilla. Para esto se construyó una grilla tridimensional en la que sus dimensiones representan: el número de iteraciones del entrenamiento (nlter), el número de muestras enviadas al optimizador en cada paso (sizeOfMinibatch) y la tasa de aprendizaje (learningRate).

- Para cada juego de hiperparámetros se entrena un modelo diferente y cada uno de ellos es evaluado en el conjunto de validación. El mejor modelo de acuerdo a la métrica MAPE es seleccionado.

- Finalmente, el mejor modelo es evaluado en el conjunto de testing utilizando la métrica MAPE.

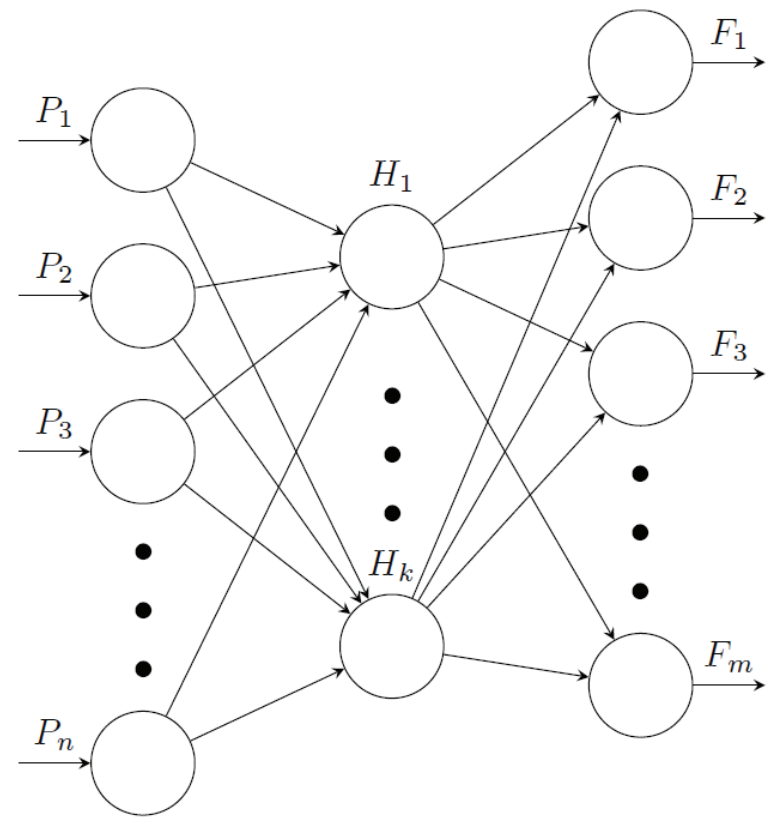

Figura 1. Arquitectura de la red neuronal entrenada.

\section{Implementación}

Para implementar la arquitectura de red neuronal presentada en la figura 1 asociada a la ecuación 4 se definieron los siguientes componentes:

- Capa de entrada, formada por 199 neuronas asociadas a cada una de las potencias de entrada.

- Capa oculta totalmente conectada, 20 neuronas a las que se les aplica la función de activación no lineal RELU (Rectified Linear Unit), para capturar los componentes no lineales del problema a resolver.

- Capa de salida, 380 neuronas representando las potencias de flujo entro los nodos desde ambos extremos. Se aplica la función de activación lineal debido a que la salida debe ser un valor continuo que puede tomar cualquier valor real. 


\section{Análisis experimental y resultados obtenidos}

En esta sección se presenta la plataforma computacional utilizada, el análisis experimental realizado y la evaluación de los resultados del caso de estudio planteado.

Plataforma computacional y software utilizado

Los modelos propuestos fueron implementados en el lenguaje de programación Python, utilizando Tensorflow. El conjunto de datos se obtiene de una base de datos Sqlite. A su vez se utilizan varias bibliotecas científicas y de aprendizaje automático para el manejo de datos, entrenamiento de modelos y visualización de resultados. El ambiente de hardware utilizado consiste en un computador de escritorio con un procesador Intel Core i7-8550U CPU@1.80GHz $1.99 \mathrm{GHz}$ de 8 núcleos y 32 GB RAM.

\section{Evaluación de resultados}

A continuación, se presentan los resultados obtenidos en el análisis del impacto del volumen de datos de entrada en este caso de estudio. Además, es reportada la evaluación del mejor modelo obtenido utilizando como modelo de referencia el Flujo DC, aplicado al mismo caso de estudio.

\section{Impacto del volumen de datos de entrada}

El tamaño del conjunto de datos de entrenamiento adecuado para el correcto aprendizaje de una red neuronal depende de la índole del problema que se quiera resolver.

Es claro que contar con un mayor volumen de datos permite entrenar un modelo con mayor precisión, ya que se posee más información. Como contrapartida, la obtención y preparación de los datos cuando el volumen es mayor tiene un costo adicional. Por otra parte, si el entrenamiento tiene un alto costo computacional, e incrementar la cantidad de datos no mejora de forma relevante la precisión, se debe considerar mantener un conjunto acotado.

Por lo tanto, el desafío radica en tener un conjunto de datos lo más compacto posible. Para decidir cuál es este conjunto, se debe encontrar un punto en dónde aumentar el tamaño del conjunto no contribuya en mejorar significativamente la calidad del modelo. Este punto se puede encontrar visualmente aplicando una estrategia conocida como elbow method. Al graficar el error respecto del tamaño del conjunto se puede observar que existe un punto de quiebre en dónde la variación del error cometido deja de ser significativa.

En este caso de estudio se entrenan varias instancias del modelo óptimo con diferentes tamaños de conjunto de datos de entrada. Cada modelo entrenado es evaluado utilizando la métrica MAPE para determinar el impacto del volumen de datos sobre la precisión obtenida.

La evaluación a través de la métrica MAPE se realizó considerando valores de potencias de flujos mayores a diferentes umbrales relevantes para los estudios. En este análisis se consideraron tres umbrales: $u_{1}=1 M V A, u_{2}=10 M V A$ у $u_{3}=50 M V A$.

El interés principal de este análisis se debe a que, en los estudios realizados en redes eléctricas para evaluar la carga de los elementos de la red, son de relevancia aquellos donde el flujo es considerable respecto de la ampacidad del elemento. 


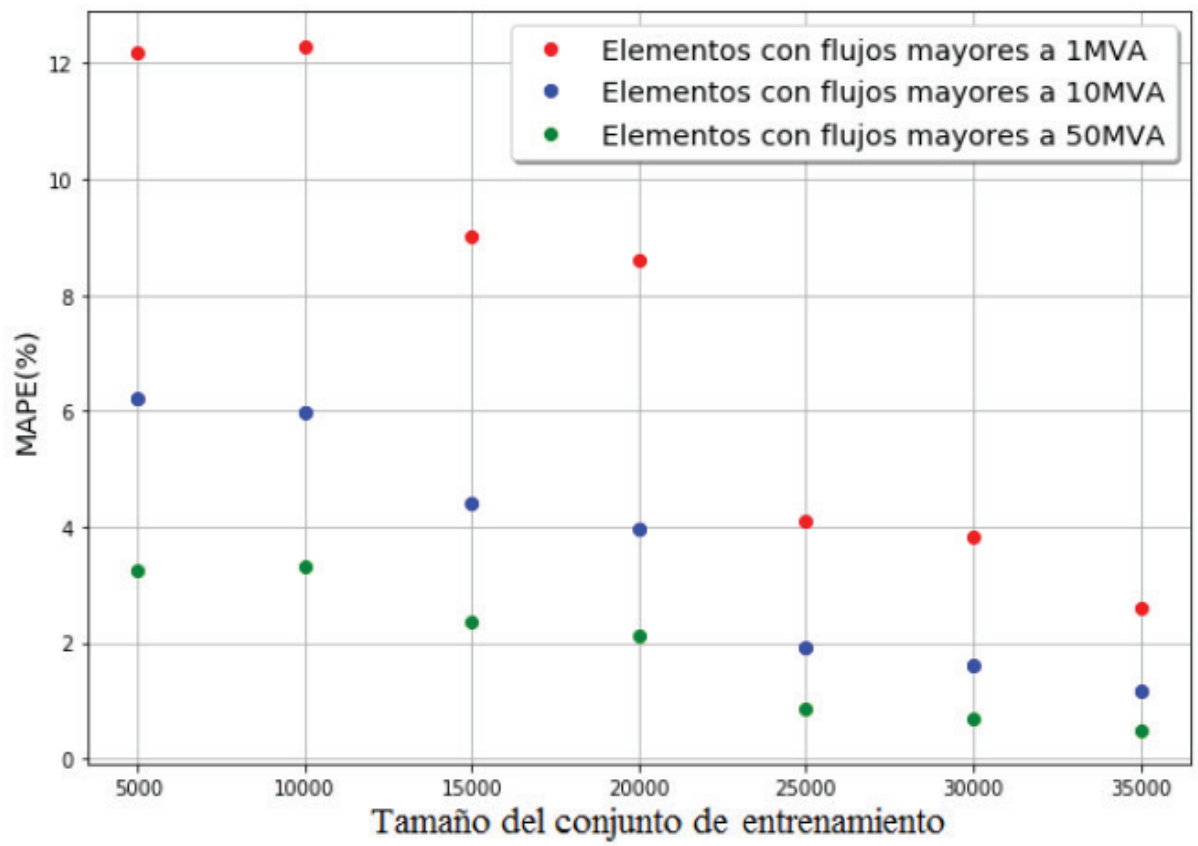

Figura 2. Evaluación MAPE según conjunto de entrenamiento.

En la figura 2 se puede confirmar como resultado relevante que incrementar la cantidad de datos mejora la precisión del modelo para todos los umbrales considerados. A su vez se observa que la evaluación considerando el umbral es aproximadamente cinco veces inferior que si se considera el umbral. Para cualquiera de los umbrales considerados, el resultado de la evaluación a través de la métrica MAPE con 35000 datos es muy bueno. Se observa además que las predicciones son mejores en elementos donde el flujo es elevado. Esto se puede explicar debido a que los grandes flujos habitualmente están asociados a demandas o generaciones directas de gran porte. En los casos de elementos de flujos menos significativos, resulta más complejo el aprendizaje por parte del modelo, ya que no están asociados a generaciones o demandas directamente.

\section{Resultados finales}

Los resultados obtenidos muestran que el modelo de red neuronal propuesto aplicado al estudio del sistema de trasmisión de Uruguay obtuvo un MAPE de $2.6 \%$. En el cuadro 1 se reportan los resultados obtenidos para el modelo desarrollado comparados con el modelo base que resuelve el Flujo DC según el trabajo de Pena [19].

Se consideran para la evaluación los valores MAPE de los tres umbrales definidos: $u_{1}=1 M V A, u_{2}=10 M V A$ y $u_{3}=50 M V A$. A la hora de comparar con el modelo base, se debe considerar que el mismo es resuelto mediante Flujo $D C$, que al despreciar las pérdidas tiene como consecuencia que el flujo es el mismo en cualquiera de los dos extremos del elemento. Para el caso de la red neuronal, se está ajustando respecto de la salida de la resolución del Flujo $A C$, por lo que se obtienen flujos diferentes en ambos extremos de cada elemento. Esto es de relevancia, ya que de forma indirecta la red neuronal aproxima las pérdidas, lo que agrega valor al modelo. 
Cuadro 1. Comparación respecto del modelo base.

\begin{tabular}{|c|c|c|c|c|}
\hline Modelo & MAPE $_{1}$ & MAPE $_{10}$ & MAPE $_{50}$ & Tiempo (ms) \\
\hline Red neuronal & 2.6 & 1.2 & 0.5 & 0.015 \\
\hline Flujo DC & 12.6 & 7.1 & 4.2 & 0.013 \\
\hline
\end{tabular}

Respecto de la comparación planteada, para el error MAPE con umbral se observa en el cuadro 1 que el modelo desarrollado reporta un valor 4.8 veces menor que el modelo base. Adicionalmente, el modelo desarrollado obtiene un valor aproximado para las pérdidas, lo que aporta valor al mismo.

En cuanto a los tiempos de ejecución, el modelo de red neuronal requiere un entrenamiento que se realiza una sola vez por topología. Para la topología considerada el tiempo de entrenamiento fue de 13 minutos.

El Flujo $D C$ no requiere entrenamiento debido a que es una aproximación analítica al problema general. Los tiempos de predicción para un sólo escenario reportados en el cuadro 1 son de 0.015 milisegundos para el modelo de red neuronal y de 0.013 milisegundos para el modelo de Flujo DC. La resolución del Flujo AC que realiza el software PSS/E para un sólo escenario tiene un tiempo de ejecución de 30 milisegundos.

Los resultados reportados en cuanto a la precisión y tiempos de ejecución para el modelo desarrollado son muy prometedores.

\section{Conclusiones y trabajo futuro}

En este artículo se abordó el problema de la resolución de flujos de carga utilizando redes neuronales y su aplicación al sistema de trasmisión eléctrico de Uruguay.

Se diseñó un modelo de red neuronal con una arquitectura en tres capas. El mismo fue entrenado utilizando técnicas de búsqueda de hiperparámetros en una grilla. El análisis experimental realizado consistió en entrenar el modelo propuesto utilizando 40000 escenarios de flujos de carga resueltos con el software PSS/E. Todos los módulos de software utilizados, se ejecutaron en un computador de escritorio con un procesador Intel Core i7-8550U CPU@1.80 GHz 1.99 $\mathrm{GHz}$ de 8 núcleos y 32 GB RAM. Para evaluar el modelo se utilizó la métrica MAPE. En primer lugar, se analizó el impacto en la calidad del modelo del volumen de datos de entrenamiento. Se constató que la utilización de mayores volúmenes de datos mejora los valores MAPE obtenidos.

Por otra parte, con el mejor modelo desarrollado se obtuvo un valor de MAPE $=2.6 \%$ considerando flujos mayores a 1MVA. Se tomó como modelo de referencia el de Flujo DC, para el cual el valor MAPE $=12.6 \%$ considerando flujos mayores a 1MVA. El tiempo de ejecución del modelo de red neuronal fue de 0.015 milisegundos por caso y la resolución del Flujo AC realizada por PSS/E ejecuta en un tiempo de 30 milisegundos por caso.

El modelo desarrollado permite resolver flujos de carga sobre una topología fija con una muy buena precisión, aproximando pérdidas y con un tiempo de ejecución adecuado. Esto implica que los estudios eléctricos realizados hoy en día se pueden enriquecer con este modelo, ya que las herramientas actuales presentan como limitante principal los tiempos de ejecución. Se puede concluir que los resultados obtenidos son muy prometedores.

Las principales líneas de trabajo futuras consisten en mejorar el modelo desarrollado de forma que aproxime tensiones y potencia reactiva. A su vez, se trabajará en incorporar como dato de entrada la definición de la topología de la red, de forma de generalizar el modelo a cualquier topología. Esta generalización implica diseñar arquitecturas más complejas de redes neuronales y utilizar herramientas informáticas de manejo de grandes volúmenes de datos. 


\section{Referencias}

[1] R. L. Sullivan, "Power system planning", McGraw-Hill Inc., New York, 1977.

[2] P. Pena, N. Morales, C. Zoppolo, A. Pizzini, y M. Artenstein, "Probabilistic power flow for the transmission system expansion planning considering distributed generation variability", CIGRE Science \& Engineering Jun. 2017, vol. 8, pp. 138 - 143, Jun. 2017.

[3] B. Stott y O. Alsac, "Fast decoupled load flow", IEEE Transactions on Power Apparatus and Systems, vol. PAS93, no. 3, pp. 859-869, 1974.

[4] K. Uemura y H. Pref, "Approximated jacobians in newton's powerflow method", in Proc. Power System Computation Conference, 1972.

[5] N. M. Peterson, W. F. Tinney, y D. W. Bree, "Iterative linear ac powerflow solution for fast approximate outage studies", IEEE Transactions on Power Apparatus and Systems, no. 5, pp. 2048-2056, 1972

[6] W.F. Tinney y J. W. Walker, "Direct solutions of sparse network equations by optimally ordered triangular factorization", Proceedings of the IEEE, vol. 55, no. 11, pp. 1801-1809, 1967.

[7] W. F. Tinney y C. E. Hart, "Power flow solution by newton's method", IEEE Transactions on Power Apparatus and systems, no. 11, pp. 1449-1460, 1967.

[8] B. Stott, J. Jardim, y O. Alsac , "Dc power flow revisited", IEEE Transactions on Power Systems, vol. 24, no. 3, pp. 1290-1300, 2009.

[9] V. Malbasa, C. Zheng, P.-C. Chen, T. Popovic, y M. Kezunovic, "Voltage stability prediction using active machine learning", IEEE Transactions on Smart Grid, vol. 8, no. 6, pp. 3117-3124, 2017.

[10] Y. Q. Chen, O. Fink, y G. Sansavini, "Combined fault location and classification for power transmission lines fault diagnosis with integrated feature extraction", IEEE Transactions on Industrial Electronics, vol. 65,no. 1, pp. 561-569, 2017.

[11] A. N. Hasan, P. P. Eboule, y B. Twala, "The use of machine learning techniques to classify power transmission line fault types and locations", 2017 International Conference on Optimization of Electrical and Electronic Equipment (OPTIM) \& 2017 Intl Aegean Conference on Electrical Machines and Power Electronics (ACEMP). IEEE, 2017, pp. 221-226.

[12] X. Pan, T. Zhao, y M. Chen, "Deepopf: Deep neural network for dc optimal power flow", 2019 IEEE International Conference on Communications, Control, and Computing Technologies for Smart Grids(SmartGridComm), 2019, pp. 1-6.

[13] A. Marot, B. Donnot, C. Romero, L. Veyrin-Forrer, M. Lerousseau, B. Donon, y I. Guyon, "Learning to run a power network challenge for training topology controllers", arXiv preprint arXiv:1912.04211, 2019.

[14] D. Owerko, F. Gama, y A. Ribeiro, "Optimal power flow using graph neural networks", ICASSP 2020-2020 IEEE International Conference on Acoustics, Speech and Signal Processing (ICASSP). IEEE, 2020,pp. 5930-5934.

[15] V. L. Paucar y M. J. Rider, "Artificial neural networks for solving the power flow problem in electric power systems", Electric Power Systems Research, vol. 62, no. 2, pp. 139-144, 2002.

[16] B. Donnot, "Deep learning methods for predicting flows in power grids: novel architectures and algorithms" Ph.D. dissertation, 2019.

[17] Siemens Industry, "Pss/e." [Online]. Available: https://new.siemens.com/global/en/products/energy/services/ transmission-distribution-smart-grid/consulting-and-planning/pss-software/pss-e.html

[18] G. Casaravilla, R. Chaer, y P. Alfaro, "Simsee: Simulador de sistemas de energía eléctrica," Proyecto PDT 47/12. Technical Report 7, Universidad de la República (Uruguay). Facultad de Ingeniería. Instituto de Ingeniería Eléctrica, Number 7-Dec, Tech. Rep., 2008.

[19] P. Pena, N.M. Morales Pignatta, A. Pizzini, y M. Artenstein, "Experiencia en la planificación de la expansión del sistema de transmisión uruguayo con gran penetración de generación renovable", Encuentro Regional Iberoamericano del CIGRE - XVII ERIAC, Ciudad del Este, Paraguay, 21-25 mayo, 2017. Disponible: https:// iie.fing.edu.uy/publicaciones/2017/PMPA17

[20] Modelo de la red de Trasmisión. UTE. Disponible: https://portal.ute.com.uy/institucional/ute/utei/mapas-ydatos-para-realizar-simulaciones-de-carga (último acceso: 29 de octubre, 2021) 